\title{
Problemas comportamentais e stress em crianças com ansiedade frente ao tratamento odontológico'
}

\author{
Children's behavioral problems and stress as a \\ consequence of dental treatment anxiety
}

\author{
Cármen Lúcia CARDOSO \\ Sonia Regina LOUREIRO ${ }^{3}$
}

\begin{abstract}
Resumo
A situação de tratamento odontológico em geral está relacionada à ansiedade e ao stress; a dor e os aspectos fisiológicos e psicológicos envolvidos no tratamento podem ser avaliados pelos pacientes, em especial pelas crianças, como potencialmente ameaçadores ao seu bem-estar. Objetivou-se comparar, quanto ao estresse infantil e ao perfil comportamental, um grupo de crianças que apresentam um alto nível de ansiedade frente ao tratamento odontológico (G1) a um grupo de crianças que colaboram com o atendimento (G2). Procedeu-se a aplicação dos instrumentos: Escala Comportamental A2 de Rutter e Escala de Estresse Infantil. As crianças do G1 apresentaram mais indicadores de estresse e de dificuldades emocionais e comportamentais que as do G2 $(p \leq 0,01)$. Conclui-se que a utilização sistemática desses instrumentos no contexto de uma clínica-escola pode permitir a identificação de crianças com dificuldades e a aplicação de medidas profiláticas e de intervenção que minimizem a ocorrência de situações de dificuldades para a realização de tratamento odontopediátrico.
\end{abstract}

Palavras-chave: ansiedade frente ao tratamento odontológico, estresse, odontopediatria, psicologia da saúde.

\begin{abstract}
Anxiety and stress are usually related to the dental treatment situation; and pain, physiological and psycological treatment issues may be evaluated specially by children patients, as they are considered well-being potential threats. The comparison between children's stress and behavioral profile of a children's group with high level of anxiety during dental treatment (G1) to a group of children, who cooperate to the attendance (G2) was this study proposal. The following technical intruments were applied: the Rutter A2 Parent Scale and the Child Stress Scale. Children from G1 had presented highier stress and behavioral difficulties rates than the G2 ( $p \leq 0.01)$ ones. According to this paper, the systematic application of these instruments in a clinical school has identified those children with difficulties, and the best prophylactic and intervention procedures that minimize the pediatric dental treatment dificulties occurrence situations.
\end{abstract}

Key words: dental treatment anxiety, stress, pediatric dental treatment; health psychology.

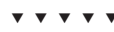

1 Artigo elaborado a partir da tese de autoria de C.L. CARDOSO, intitulada"Tratamento odontopediátrico no contexto de uma clínica-escola: avaliação do estresse da criança, do acompanhante e do aluno. Programa de Pós-Graduação em Psicologia da FFCLRP-USP, 2002. Pesquisa financiada pela FAPESP. 2 Departamento de Psicologia e Educação, Faculdade de Filosofia Ciências e Letras de Ribeirão Preto, Universidade de São Paulo. Rodovia Bandeirantes, 3900, 14040-901, Ribeirão Preto, SP, Brasil. Correspondência para/Correspondence to: C.L. CARDOSO. E-mail: <carmen@ffclrp.usp.br>.

3 Departamento de Neurologia, Psiquiatria e Psicologia Médica, Faculdade de Medicina de Ribeirão Preto, Universidade de São Paulo. Ribeirão Preto, SP, Brasil. 
As intervenções odontológicas freqüentemente provocam reações negativas por parte das crianças; segundo alguns autores (Giron, 1982; Silva, Guedes Pinto, Reginato \& Chippari, 1992; Torriane, 1999), tais reações podem ser associadas à importância da boca como fonte de gratificação nos primeiros anos de vida.

Corkey e Freeman (1994) afirmam que por volta dos seis ou sete anos há uma diminuição da ansiedade frente à situação odontológica, havendo um maior número de crianças que enfrentam sem dificuldade essa situação. Os autores relacionam a diminuição da ansiedade à fase de desenvolvimento psicológico em que se encontram essas crianças, ou seja, já apresentam uma maior independência dos pais, têm boas relações com grupos e pares, bom controle do intestino e da bexiga e dormem durante toda a noite. Apontam, através de estudo sistemático com crianças nessa faixa etária, que altos índices de ansiedade em relação ao tratamento odontológico em geral estão relacionados a atrasos no desenvolvimento psicológico.

A situação de tratamento odontológico, segundo Eli (1992), está freqüentemente relacionada à ansiedade e ao stress. Existem estímulos fisiológicos, como, por exemplo, a dor, e aspectos psicológicos envolvidos no tratamento dentário que podem ser avaliados pelos pacientes como potencialmente ameaçadores ao seu bem-estar.

Jacob (1998) afirma que o stress vivenciado pelo paciente amplia o seu medo e a sua percepção da dor, diminuindo a sua capacidade de colaborar com o tratamento.

A criança, tal qual o adulto, está susceptível ao estresse. A vivência de um stress intenso pode gerar graves conseqüências para a criança. Lipp, Souza, Romano e Covolan (1991) apontam como conseqüências do estresse infantil a presença de doenças físicas, como asma, alergias e distúrbios dermatológicos, que podem ocorrer como resultado do esforço que o organismo faz para lidar com o estresse. Também podem ocorrer desajustes psicológicos, tais como comportamento agressivo, enurese, gagueira, medo exagerado quando submetido à situação de estresse, e ainda dificuldades de relacionamento.

Em relação à atenção odontológica, o estresse e a ansiedade têm sido relacionados com a evitação ou fuga do tratamento dentário, representando uma barreira à utilização desses serviços, mesmo quando muito necessários, comprometendo a saúde bucal do indivíduo (Cesar, Moraes, Milgrom \& Kleinknecht, 1993; Eli, Uziel, Bath \& Kleinhauz, 1997; Elter, Strauss \& Beck 1997; Rosa \& Ferreira, 1997; Slovin, 1997).

Segundo observa Giron (1988), os pacientes pediátricos não têm opção de escolha e são geralmente levados pelos pais para o tratamento dentário. Tais pacientes em geral manifestam o seu medo através de seu comportamento, como, por exemplo, choro, recusa em abrir a boca, chute, vômitos, na tentativa de evitar o atendimento odontológico.

O desencadeamento da ansiedade odontológica tem sido relacionado a fatores referentes a aspectos comportamentais e de personalidade dos pacientes, à própria situação de tratamento e à aprendizagem através da observação ou imitação de modelos (Eli et al., 1997). Entre esses fatores, a importância das primeiras relações com o dentista é ressaltada por Possobon, Caetano e Moraes (1998). Esses autores descrevem que "as experiências odontológicas iniciais deveriam ocorrer com um mínimo de trauma físico e psicológico" (p.80). Milgrom, Weinstein e Getz (1995) afirmam que um dos grandes objetivos do tratamento odontopediátrico é desenvolver atitudes positivas nas crianças frente a essa situação, uma vez que é mais difícil e mais caro alterar comportamentos ansiosos.

A importância dos cuidados com os aspectos emocionais e afetivos das crianças durante o atendimento odontológico é enfatizada por Silva et al. (1992). Esses autores salientam que contribuições da psicologia e da educação podem ampliar os benefícios do tratamento odontológico e evitar o aparecimento de traumas psicológicos. Giron (1988) aponta que "...qualquer programa de saúde oral comunitário estará incompleto e será, portanto, ineficaz, se não levar em conta, também, os fatores emocionais envolvidos" (p.125).

Nesse contexto, o objetivo do presente estudo é comparar um grupo de crianças sem necessidades especiais que apresentam um alto nível de ansiedade frente ao tratamento odontológico, necessitando de contenção física para a realização do mesmo, a um grupo de crianças que colaboram com o tratamento odontológico. Para tal avaliaram-se o stress infantil e o perfil comportamental das crianças de ambos os grupos. 


\section{Método}

O presente estudo foi desenvolvido junto à clínica-escola da Faculdade de Odontologia de Ribeirão Preto, Universidade de São Paulo (FORP/USP) após a aprovação do Comitê de Ética (Processo n¹012299). O estudo foi desenvolvido nas condições naturais de atendimento da clínica. A medida de contenção física é utilizada na clínica de odontopediatria, com consentimento por escrito dos pais ou responsáveis, quando a criança não permite a execução dos procedimentos odontológicos.

\section{Participantes}

Das 100 crianças inicialmente avaliadas, foram identificadas, com base nos dados observacionais das seis sessões iniciais de atendimento, 27 crianças que necessitaram de contenção física para a realização do tratamento odontológico. Definiu-se como critério de exclusão: crianças com atraso no desenvolvimento cognitivo (avaliado através de teste específico), com má formação congênita, com problemas neurológicos e ou psicológicos já diagnosticados ou tratados, com internações hospitalares por problemas de saúde e que não residam com a família biológica.

Das 27 crianças inicialmente identificadas, selecionou-se 20 que preenchiam os critérios e que requereram contenção física apresentando ansiedade intensa frente ao tratamento odontológico, não colaborando com o atendimento (Grupo 1). Com base nas características demográficas quanto à idade e sexo dessas crianças, selecionaram-se os 20 participantes do grupo de crianças que colaboraram com o atendimento, ou seja, que aceitaram os procedimentos odontológicos propostos.

A faixa etária dos participantes ia de seis a onze anos e cinco meses, sendo incluídos em cada grupo onze do sexo masculino e nove do sexo feminino.

Todas as crianças incluídas no estudo foram submetidas a tratamento odontológico envolvendo procedimentos técnicos, segundo a avaliação sistemática de odontopediatras, pouco invasivos $(G 1=30,3 \%, G 2=31,1 \%$ - profilaxia) e invasivos ( $\mathrm{G} 1=69,7 \%$ e G2=68,9\% - restaurações e endodontia), não tendo sido observada diferença significativa entre os grupos quanto ao nível de invasão (Teste do Qui Quadrado, $p \leq 0,05$ )

\section{Instrumentos para a coleta de dados}

- Escala de Stress Infantil (ESI): utilizada para a avaliação do estresse infantil e do tipo de reações mais freqüentes, ou seja: físicas, psicológicas, psicológicas com componente depressivo e psicofisiológicas. Contém 35 itens em uma escala do tipo likert de cinco pontos. Foi validada por Lipp e Lucarelli (1998) e a análise fatorial evidenciou os quatro fatores acima descritos e um fator geral - stress. Refere bons indicadores de consistência interna (coeficiente alfa de Cronbach 0,90).

- Escala Comportamental Infantil A2 de Rutter: utilizada para a avaliação de problemas comportamentais e emocionais das crianças, adaptada por Graminha (1994). Contém 36 itens distribuídos em três tópicos, que são: problemas de saúde (oito itens), hábitos (sete itens) e comportamento. O respondente deve assinalar a freqüência ou a intensidade do problema apresentado. Possui índices psicométricos adequados, com valores de fidedignidade (teste reteste) aceitáveis para 97\% dos itens da escala adaptada e bons indicadores de validade discriminante na identificação de casos que necessitam de ajuda psicológica.

Todas as crianças foram avaliadas individualmente, em uma única sessão de aproximadamente 25 minutos. As mães foram entrevistadas sobre os comportamentos das crianças individualmente, em situação de entrevista face-a-face, em uma única sessão, com duração média de aproximadamente 20 minutos.

As técnicas utilizadas foram codificadas de acordo com as proposições técnicas. Procedeu-se ao tratamento estatístico não paramétrico dos dados comparando-se os grupos com relação aos aspectos comportamentais e ao estresse. Para a comparação foram utilizados o Teste Mann Whitney, Teste do Qui Quadrado e/ou Teste Exato de Fisher.

Para todos os testes estatísticos utilizados adotou-se como critério valores de $p \leq 0,05$.

\section{Resultados}

Os resultados obtidos por meio da Escala Comportamental Infantil A2 de Rutter, relativos a 
aspectos comportamentais das crianças dos dois grupos, segundo a avaliação das mães, são apresentados na Tabela 1.

Observou-se diferenças estatisticamente significativas entre os dois grupos de crianças no que se refere aos resultados relativos ao escore total e aos escores de saúde e comportamento. Constatou-se que as crianças do Grupo 1 apresentaram indicadores que revelaram maior freqüência de problemas comportamentais em comparação com as do Grupo 2.

Os dados normativos da Escala sinalizam como nota de corte o escore total acima de 16 pontos, considerado sugestivo da necessidade de atendimento psicológico ou psiquiátrico da criança (Graminha \& Coelho, 1994). As porcentagens de crianças de cada grupo que atingiram a nota de corte, bem como a comparação entre os grupos, encontram-se na Tabela 2.

Comparativamente ao Grupo 2, o Grupo 1 apresentou porcentagem significativamente maior de crianças com resultados acima da nota de corte, sugestivos da necessidade de encaminhamento para atendimento especializado.
Os resultados obtidos em cada um dos itens específicos da Escala Comportamental Infantil A2 de Rutter e a comparação entre os grupos e com os resultados do estudo normativo de Graminha e Coelho (1994) são apresentados na Tabela 3.

Observou-se que em três itens da categoria comportamento os resultados do Grupo 1 foram significativamente maiores que os do Grupo 2, a saber: destrói as suas coisas e as dos outros; briga freqüentemente; criança difícil, complicada ou muito particular.

Quando comparados aos dados normativos, verifica-se que em 12 itens o Grupo 1 obteve resultados maiores que o normativo, a saber: em um item da categoria saúde (asma e crises respiratórias), em dois itens da categoria hábitos (dificuldade de fala, gagueira) e em nove itens da categoria comportamento (agitada; impaciente; destrói as suas coisas e as dos outros; irritável; chupa dedos; criança difícil, complicada ou muito particular; maltrata crianças, agarrada à mãe e insegura). O Grupo 2 apresentou resultado maior que o esperado pela norma em um item da categoria comportamento (agarrada à mãe).

Em três itens, os Grupos 1 e 2 apresentaram resultados menores que o normativo. As crianças do

Tabela 1. Escala Comportamental Infantil A2 de Rutter. Média (M), Desvio-Padrão (DP) e Mediana (ME) dos escores obtidos pelas crianças dos Grupos 1 e 2.

\begin{tabular}{|c|c|c|c|c|c|c|c|c|c|}
\hline \multirow{2}{*}{ Escala } & \multicolumn{4}{|c|}{1} & \multicolumn{3}{|c|}{2} & \multirow[b]{2}{*}{$p$} & \multirow[b]{2}{*}{ Comparação } \\
\hline & M & \pm & DP & ME & M & $\pm \mathrm{DP}$ & ME & & \\
\hline Escore Total & 22,75 & \pm & 11,62 & 22,50 & 12,95 & $\pm 8,00$ & 10,50 & 0,01 & $1>2^{* *}$ \\
\hline Saúde & 3,50 & \pm & 2,50 & 3,00 & 2,00 & $\pm 1,72$ & 2,00 & 0,05 & $1>2^{*}$ \\
\hline Hábitos & 2,05 & \pm & 2,16 & 1,50 & 1,50 & $\pm 1,19$ & 1,00 & 0,08 & $1=2$ \\
\hline Comportamento & 17,20 & \pm & 8,45 & 17,00 & 9,90 & $\pm 6,38$ & 8,00 & 0,01 & $1>2^{* *}$ \\
\hline
\end{tabular}

${ }^{*} p \leq 0,05 ;{ }^{*} p \leq 0,01$. Teste Mann Whitney.

Tabela 2. Escala Comportamental Infantil A2 de Rutter. Freqüência (F) e porcentagem (\%) de crianças dos Grupos 1 e 2 que apresentaram indicadores superiores ou inferiores à nota de corte, segundo os critérios propostos pelos dados normativos (Graminha \& Coelho, 1994).

\begin{tabular}{|c|c|c|c|c|c|c|}
\hline \multirow{2}{*}{ Rutter } & \multicolumn{2}{|c|}{1} & \multicolumn{2}{|c|}{2} & \multirow[b]{2}{*}{$p$} & \multirow[b]{2}{*}{ Comparação } \\
\hline & $\mathrm{F}$ & $\%$ & $\mathrm{~F}$ & $\%$ & & \\
\hline Presença & 13 & 65 & 4 & 20 & 0,01 & $1>2^{*}$ \\
\hline Ausência & 7 & 35 & 16 & 80 & & \\
\hline
\end{tabular}

$8 \quad * p \leq 0,01$. Teste Qui-Quadrado. 
Grupo 1 apresentaram valores menores que os normativos em dois itens da categoria hábitos, ou seja, dificuldade de alimentação e de sono. E as crianças do Grupo 2 apresentaram valores menores que o normativo em dois itens, a saber: dificuldade de sono da categoria hábitos e criança fechada e solitária incluído na categoria comportamento.
Os resultados relativos às manifestações de estresse para cada grupo de crianças são apresentados na Tabela 4.

Observa-se que as crianças do Grupo 1 apresentaram escore total significativamente maior que as do Grupo 2, indicando a presença de maior número de respostas de estresse no Grupo 1.

Tabela 3. Escala Comportamental Infantil A2 de Rutter. Frequência (F) e Porcentagens das respostas das crianças dos Grupos 1 e 2 aos itens e comparação com os resultados do estudo normativo (N).

\begin{tabular}{|c|c|c|c|c|c|c|}
\hline \multirow{2}{*}{ Itens da escala Grupos } & \multicolumn{2}{|c|}{$1(n=20)$} & \multicolumn{2}{|c|}{$2(n=20)$} & \multirow{2}{*}{$\frac{(n=1614)}{1}$} & \multirow{2}{*}{$\begin{array}{c}\text { Comparação } \\
\text { Entre G1,G2 e N }\end{array}$} \\
\hline & $\mathrm{F}$ & $\%$ & $\mathrm{~F}$ & $\%$ & & \\
\hline \multicolumn{7}{|l|}{ Saúde } \\
\hline Dor de cabeça & 10 & 0,50 & 8 & 0,40 & 0,44 & \\
\hline Dor de estômago & 6 & 0,30 & 5 & 0,25 & 0,24 & \\
\hline Asma, crises respiratórias & 7 & 0,35 & 6 & 0,30 & 0,13 & $\mathrm{G} 1>N^{*}$ \\
\hline Enurese & 4 & 0,20 & 1 & 0,05 & 0,13 & \\
\hline Encoprese & 0 & 0 & 1 & 0,05 & 0,02 & \\
\hline Mau humor & 16 & 0,80 & 12 & 0,60 & 0,59 & \\
\hline Recusa a escola & 4 & 0,20 & 1 & 0,05 & 0,10 & \\
\hline "Mata" aula & 0 & 0 & 0 & 0 & 0,06 & \\
\hline \multicolumn{7}{|l|}{ Hábitos } \\
\hline Dificuldade de fala & 4 & 0,20 & 1 & 0,05 & 0,07 & $\mathrm{G} 1>\mathrm{N}^{*}$ \\
\hline Gagueira & 5 & 0,25 & 1 & 0,05 & 0,08 & $\mathrm{G} 1>\mathrm{N}^{*}$ \\
\hline Roubo & 3 & 0,15 & 1 & 0,05 & 0,08 & \\
\hline Dificuldade de alimentação & 5 & 0,25 & 7 & 0,35 & 0,48 & $\mathrm{G} 1<\mathrm{N}^{*}$ \\
\hline Dificuldade de sono & 4 & 0,20 & 1 & 0,05 & 0,47 & $\mathrm{G} 1<\mathrm{N}^{*}, \mathrm{G} 2<\mathrm{N}^{*}$ \\
\hline Medo & 11 & 0,55 & 6 & 0,30 & 0,47 & \\
\hline Movimentos repetitivos & 4 & 0,20 & 1 & 0,05 & 0,11 & \\
\hline \multicolumn{7}{|l|}{ Comportamento } \\
\hline Agitado & 14 & 0,70 & 12 & 0,60 & 0,46 & $\mathrm{G} 1>\mathrm{N}^{*}$ \\
\hline Impaciente & 16 & 0,80 & 12 & 0,60 & 0,42 & $\mathrm{G} 1>N^{*}$ \\
\hline Destrói coisas & 13 & 0,65 & 4 & 0,20 & 0,26 & $\mathrm{G} 1>\mathrm{G} 2 *, \mathrm{G} 1>\mathrm{N}^{*}$ \\
\hline Briga com crianças & 11 & 0,55 & 4 & 0,20 & 0,38 & $\mathrm{G} 1>\mathrm{G} 2 *$ \\
\hline Não querida por pares & 2 & 0,10 & 4 & 0,20 & 0,24 & \\
\hline Muito preocupada & 13 & 0,65 & 13 & 0,65 & 0,49 & \\
\hline Fechada & 5 & 0,25 & 1 & 0,05 & 0,25 & $\mathrm{G} 2<\mathrm{N}^{*}$ \\
\hline Irritável & 15 & 0,75 & 9 & 0,45 & 0,51 & $\mathrm{G} 1>N^{*}$ \\
\hline Tristonha, infeliz & 8 & 0,40 & 3 & 0,15 & 0,23 & \\
\hline Chupa dedo & 5 & 0,25 & 3 & 0,15 & 0,08 & $\mathrm{G} 1>\mathrm{N}^{*}$ \\
\hline Rói unha & 4 & 0,20 & 3 & 0,15 & 0,25 & \\
\hline Desobediente & 17 & 0,85 & 14 & 0,70 & 0,69 & \\
\hline Dificuldade de concentração & 11 & 0,55 & 7 & 0,35 & 0,44 & \\
\hline Medo de situações novas & 5 & 0,25 & 4 & 0,20 & 0,33 & \\
\hline Criança difícil & 10 & 0,50 & 3 & 0,15 & 0,25 & $\mathrm{G} 1>\mathrm{G} 2^{*}, \mathrm{G} 1>\mathrm{N}^{*}$ \\
\hline Mentirosa & 11 & 0,55 & 9 & 0,45 & 0,40 & \\
\hline Maltrata crianças & 8 & 0,40 & 3 & 0,15 & 0,16 & $\mathrm{G} 1>\mathrm{N}^{*}$ \\
\hline Fala palavrões & 9 & 0,45 & 4 & 0,20 & 0,27 & \\
\hline Agarrada à mãe & 15 & 0,75 & 17 & 0,85 & 0,50 & $\mathrm{G} 1>\mathrm{N}^{*}, \mathrm{G} 2>\mathrm{N}^{*}$ \\
\hline Tímida & 13 & 0,65 & 11 & 0,55 & 0,50 & \\
\hline Insegura & 7 & 0,35 & 2 & 0,10 & 0,31 & \\
\hline
\end{tabular}

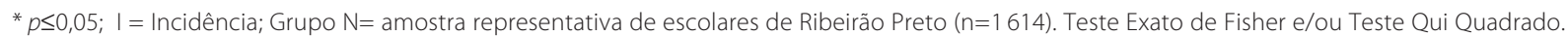


Tabela 4. Escala de Stress Infantil. Média (M), Desvio-Padrão (DP) e Mediana (ME) dos escores obtidos pelas crianças dos Grupos 1 e 2.

\begin{tabular}{|c|c|c|c|c|c|c|c|c|c|}
\hline \multirow{2}{*}{ Escala } & \multicolumn{4}{|c|}{1} & \multicolumn{3}{|c|}{2} & \multirow[b]{2}{*}{$p$} & \multirow[b]{2}{*}{ Comparação } \\
\hline & $M$ & \pm & $\mathrm{DP}$ & $\mathrm{ME}$ & $M$ & $\pm \mathrm{DP}$ & ME & & \\
\hline Escore Total & 53,45 & \pm & 21,65 & 53,00 & 34,68 & $\pm 17,28$ & 38,00 & 0,01 & $1>2^{*}$ \\
\hline
\end{tabular}

${ }^{*} p \leq 0,01$. Teste Mann Whitney.

\section{Discussão}

Verificou-se que tanto as crianças que necessitaram de contenção física quanto as que colaboraram com o atendimento odontológico foram submetidas a tratamentos odontológicos com níveis de invasão comparável do ponto de vista técnico, segundo a avaliação dos profissionais. Nesse sentido, pode-se supor que as manifestações de ansiedade apresentadas pelas crianças que requereram contenção física (Grupo 1) durante o tratamento odontológico não estão diretamente relacionadas ao nível de invasão dos procedimentos.

Analisando-se os resultados relativos à Escala Comportamental Infantil - A2 de Rutter, respondida pelas mães, verificou-se que as crianças dos dois grupos diferenciaram-se em relação ao escore total e às queixas relativas a dificuldades quanto às áreas saúde e comportamento.

As crianças que necessitaram de contenção física para a realização do tratamento odontológico apresentaram mais indicadores de problemas comportamentais que as crianças que colaboraram com o atendimento odontológico. Em relação à nota de corte proposta por Graminha e Coelho (1994), observou-se uma maior porcentagem de crianças do Grupo 1 com resultados indicativos de problemas ou dificuldades de ordem emocional. Pode-se pensar que essas crianças ao experimentarem um aumento da ansiedade situacional expressaram-na diretamente no ambiente através de comportamentos que dificultam ou impedem o tratamento odontológico.

Ao analisar as dificuldades comportamentais e emocionais apresentadas pelas crianças que necessitaram de contenção física, pode-se pensar que essas estão enfrentando dificuldades quanto ao seu desenvolvimento, pois, segundo Corkey e Freeman (1994), altos níveis de ansiedade odontológica em criança com mais de seis anos estão em geral relacionados a atraso no desenvolvimento psicológico.
As crianças que não colaboraram com o atendimento odontopediátrico apresentaram maior freqüência nos itens: asma ou crises respiratórias, dificuldade de fala, gagueira, chupar dedo, ser uma criança muito agarrada à mãe e insegura, comparativamente ao grupo normativo de Graminha e Coelho (1994). Segundo Lipp et al. (1991), esses indicadores estão geralmente associados ao estresse vivenciado pela criança. Pode-se apresentar como hipótese, ainda, que para o enfrentamento de situações de dificuldade essas crianças tentem conter os próprios sentimentos, deslocando-os para o corpo e apresentando comportamentos sugestivos de imaturidade.

Por outro lado, essas crianças comparativamente ao grupo normativo apresentaram também maior freqüência de comportamentos nos itens: agitada, impaciente, destrói coisas, irritável, difícil, maltrata outras crianças, sinalizando a emissão de comportamentos de exteriorização dos sentimentos, ou seja, a ansiedade experimentada é depositada no ambiente.

Segundo Lipp et al. (1991), as crianças que experimentam estresse intenso apresentam comportamentos agressivos como forma de alívio e redução da ansiedade, o que pode minimizar o estresse do momento, caracterizando, assim, um comportamento reforçado pelas conseqüências que produz.

Com relação à situação que envolve o tratamento odontológico, Silva et al. (1992) afirmam que, para a criança, o profissional (cirurgião dentista) e o ambiente do consultório são sentidos como repulsivos. Pode-se supor que o tratamento odontológico é sentido como uma situação aversiva para as crianças que não colaboram com o atendimento e que, segundo a avaliação das mães, apresentam maiores indicadores de problemas comportamentais e emocionais. 
As crianças que colaboraram com o tratamento odontológico apresentaram, segundo a avaliação das mães, menos indicadores de problemas comportamentais e emocionais. Comparativamente aos valores normativos, essas crianças apresentaram menor freqüência de comportamentos no item criança fechada e solitária, o que também sugere maior sociabilidade por parte dessas crianças.

Os resultados referentes à avaliação do estresse mostraram que os dois grupos diferenciaram-se quanto às manifestações presentes na Escala de Stress Infantil. Observou-se que as crianças que necessitaram de contenção física apresentaram mais indicadores de estresse que as que colaboraram com tratamento odontopediátrico. Pode-se supor que as crianças que necessitaram de contenção física, apresentando mais indicadores de estresse, vivenciaram a situação de atendimento odontológico como muito ameaçadora, o que segundo Jacob (1998) pode diminuir a capacidade delas de colaborarem com o tratamento. Tal dado corrobora também a afirmação de Eli (1992), que considera a situação de tratamento odontológico como potencialmente estressante.

Selye (1956) afirma que, ao se deparar com um estressor, o organismo se prepara para reações de luta e fuga. Esse autor considera que a adaptabilidade do corpo é finita e aponta a necessidade de minimizar situações estressantes, poupando com isso as reservas de energia do sujeito. Para tanto, Selye (1983) sugere que se diminua a possibilidade de que um evento neutro se torne estressante, que sejam suprimidos os estressores desnecessários à vida e que se desenvolvam habilidades para o enfrentamento de condições adversas.

A análise dos resultados obtidos neste estudo chama a atenção para a necessidade de planejar modelos de intervenção que favoreçam o tratamento odontopediátrico de crianças com mais indicadores de estresse de modo a favorecer-Ihes um melhor enfrentamento dessa situação.

\section{Considerações Finais}

O perfil das crianças que necessitaram de contenção física caracterizou-se pela presença de comportamentos pouco socializados com elevadas manifestações de estresse, dificultando a aceitação e adaptação frente ao tratamento odontológico. O perfil das crianças que colaboraram com o atendimento odontológico caracterizou-se pela presença de sinais de maior sociabilidade, com menos indicadores de estresse.

Analisando-se alguns limites em relação aos aspectos metodológicos deste estudo, pode-se considerar que a faixa etária estudada foi ampla. Por tratar-se de um estudo desenvolvido no contexto natural de aplicação, sem qualquer interferência na seleção de crianças para o atendimento odontológico, não foi possível restringir a faixa etária. Por outro lado, ao serem priorizados os critérios desempenho cognitivo pelo menos nível médio inferior, ausência de problemas psicológicos e/ou neurológicos diagnosticados e/ou tratados, ausência de história prévia de internação hospitalar por problemas de saúde, de certo modo procurou-se garantir certos padrões normativos com relação às crianças da amostra. Com a observação desses critérios, considera-se que foi possível controlar a presença de indicadores de ansiedade que poderiam ter relação com outros fatores que não a situação de tratamento odontológico.

Nesse contexto de atendimento odontológico a crianças pouco colaboradoras em uma clínica-escola, fazem-se necessários novos estudos que acompanhem o atendimento ao longo das suas diferentes etapas, com mais recursos de observação do processo e das interações.

Considera-se que a utilização sistemática desses instrumentos no contexto de uma clínica-escola poderá prevenir e/ou minimizar a ocorrência de situações de impasse, como a que envolve a contenção física de crianças sem necessidades especiais para a realização de tratamento odontológico, em contexto de aprendizagem e formação, favorecendo o planejamento de intervenções conjuntas da psicologia e da odontologia.

\section{Referências}

Cesar, J., Moraes, A.B.A., Milgrom, P., \& Kleinknecht, R.A. (1993). Cross validation of a Brazilian version of the Dental Fear Survey. Community Dentistry and Oral Epidemiol, 21 (1), 148-50. 
Corkey, B., \& Freeman, R. (1994). Predictors of dental anxiety in sixyear old children: Findings from a pilot study. Journal of Dentistry for Children, 61 (4), 267-71.

Eli, L. (1992). Oral Psychophysiology: Stress, pain and behavior in dental care. Florida: CRC Press.

Eli, L., Uziel, N., Bath, R., \& Kleinhauz, M. (1997). Antecedents of dental anxiety: Learned responses versus personality traits. Community Dentistry and Oral Epidemiology, 25 (3), 233-7.

Elter, J.R., Strauss, R.P., \& Beck, J. (1997). Assessing dental anxiety, dental care use and oral status in older adults. Journal American Dental Association, 128 (5), 591-7.

Giron, M.C.C. (1982). O cirurgião dentista aos olhos da criança. Revista de Psiquiatria do Rio Grande do Sul, 4 (1), $38-45$

Giron, M.C.C. (1988). Fundamentos psicológicos da prática odontológica (p.125). Porto Alegre: D.C. Luzzatto Editora.

Graminha, S.S.V. (1994). A escala comportamental infantil de Rutter A2: estudos de adaptação e fidedignidade. Estudos de Psicologia, 11 (3), 34-42.

Graminha, S.S.V., \& Coelho, W.F. (1994). Problemas emocionais e comportamentais em crianças que necessitam ou não de atendimento psicológico ou psiquiátrico [Resumos]. In Anais da XXIV Reunião Anual de Psicologia (p.258). Ribeirão Preto: Sociedade Brasileira de Psicologia.

Jacob, L.S. (1998). Psicologia e odontologia. In B. Rangé (Org.). Psicoterapia Comportamental e Cognitiva. São Paulo: Editorial Psy.

Lipp, M.E.N., \& Lucarelli, M.D.M. (1998). Escala de stress infantil - ESI - manual. São Paulo: Casa do Psicólogo.
Lipp, M.E.N., Souza, E.A.P., Romano, A.S.P.F., \& Covolan, M.A. (1991). Como Enfrentar o Stress Infantil. São Paulo: Ícone Editora.

Milgrom, P., Weinstein, P., \& Getz, T. (1995).Treating fearful dental patients, Washington: University of Washington in Seattle.

Possobon, R.F., Caetano, M.E.S., \& Moraes, A.B.A. (1998). Odontologia para crianças não colaboradoras: relato de casos. Revista Brasileira de Odontologia, 55 (2), 80-3.

Rosa, A.L., \& Ferreira, C.M. (1997). Ansiedade odontológica: nível de ansiedade, prevalência e comportamento dos indivíduos ansiosos. Revista Odontológica Brasileira, 54 (3), 171-4.

Selye, H.A. (1956). The stress of life. New York: Longmans.

Selye, H.A. (1983).The Stress Concept: Past, present, and future. In C.L. Cooper (Org.). Stress research: Issues for the eighties (pp.1-20). New York: John Wiley \& Sons.

Silva, S.R.G., Guedes Pinto, A.C., Reginato, S.M., \& Chippari, M. (1992). A percepção da criança com relação a odontopediatria. Um acréscimo da psicologia a odontopediatria. Revista de Odontopediatria, 1 (3), 127-55.

Slovin, M. (1997). Managing the anxious and phobic dental patient. The New York State Dental Journal, 63 (7), $36-40$

Torriane, D.D. (1999). Análise do comportamento de bebês durante atendimento odontológico: relação entre sexo, idade e dentes irrompidos. Dissertação de mestrado não publicada, Pós-Graduação em Odontope-diatria, Universidade Estadual Paulista. Araçatuba, SP.

Recebido para publicação em 26 fevereiro de 2004 e aceito em 16 de fevereiro de 2005. 